\title{
Contrast Staining may be Associated with Intracerebral Hemorrhage but Not Functional Outcome in Acute Ischemic Stroke Patients Treated with Endovascular Thrombectomy
}

\author{
Hong An ${ }^{1,2, \#, ~ W e n b o ~ Z h a o, " \#, ~ J i a n g u o ~ W a n g ~}{ }^{3, \#, ~ J o s h u a ~ C . ~ W r i g h t ~}{ }^{4}$, Omar Elmadhoun ${ }^{5,6}$, Di
} $\mathbf{W u}^{2}$,Shuyi Shang ${ }^{1}$, Chuanjie Wu${ }^{1}$, Chuanhui $\mathbf{L i}^{7}$, Longfei Wu ${ }^{1,2}$, Jian Chen ${ }^{8}$, Jiangang Duan ${ }^{7}$, Hongqi Zhang ${ }^{8}$, Haiqing Song1, Yuchuan Ding ${ }^{2,5}$, Xunming $\mathbf{J i}^{2,8, *}$

\begin{abstract}
${ }^{1}$ Department of Neurology, Xuanwu Hospital, Capital Medical University, Beijing, China. ${ }^{2}$ China-America Institute of Neuroscience, Xuanwu Hospital, Capital Medical University, Beijing, China. ${ }^{3}$ Department of Rehabilitation, Beijing Tongren Hospital, Capital Medical University, Beijing, China. ${ }^{4}$ Wayne State University School of Medicine, Detroit, MI, USA. ${ }^{5}$ Department of Neurosurgery, Wayne State University School of Medicine, Detroit, MI, USA. ${ }^{6}$ Department of Anesthesiology, Critical Care, and Pain Medicine, Beth Israel Deaconess Medical Center/Harvard Medical School, Massachusetts, USA. ${ }^{7}$ Department of Emergency, Xuanwu Hospital, Capital Medical University, Beijing, China. ${ }^{8}$ Department of Neurosurgery, Xuanwu Hospital, Capital Medical University, Beijing, China
\end{abstract}

[Received May 11,2018; Revised Jul 29, 2018; Accepted August 7, 2018]

\begin{abstract}
To evaluate the incidence of post-interventional contrast staining (PICS) in acute ischemic stroke (AIS) Chinese patients who were treated with endovascular thrombectomy (ET) and investigate potential association of PICS with functional outcome and intracerebral hemorrhage (ICH). This observational study was based on a single-center prospective registry study. AIS patients who underwent ET from January 2013 to February 2017 were recruited into this study. All patients had dual-energy CT (DECT) scan of the head at 12 to 24 hours post-ET. The primary outcome was the incidence of PICS. Secondary outcomes were total ICH, symptomatic ICH (sICH), 3-month functional outcome, and long-term functional outcome. One hundred and eighty patients were enrolled in this study. PICS was detected in 50 patients $(28 \%)$ based on the postinterventional CT scan. We first used basic statistical analyses, showing that the incidence of both total ICH $(60 \%$ vs. $25 \%$, p<0.001) and sICH $(18 \%$ us. $8 \%, p=0.044)$ were higher in patients with PICS than those without, and fewer patients achieved no disability $(\mathrm{mRS} \leq 1)$ in the PICS group compared to the control group at both 3 -month and long-term follow-up ( $<<0.01$ each). Howe ver, multivariate regression analysis further revealed that PICS only increased total (adjusted odds ratio, 7.38; $95 \%$ confidence interval 1.66 to $32.9 ; \mathrm{p}=0.009$ ) but not $\mathrm{sICH}$ risk. Furthermore, the logistic regression analyses did not show statistical difference in good clinical outcomes or mortality between the two groups. PICS is a common phenomenon in Chinese AIS patients. It is associated with total ICH after ET, but it seems to have no effect on functional outcome and sICH. Further large-scale studies are warranted to validate these results.
\end{abstract}

Key words: post-interventional contrast staining, endovascular thrombectomy, outcome, intracerebral hemorrhage

Post-interventional contrast staining (PICS) is a common phenomenon in acute ischemic stroke (AIS) patients after endovascular thrombectomy (ET) and it usually resolves within 24-48 hrs [1,2]. However, it is still unclear whether

* Corresponding Author: Dr. Xunming Ji, China-America Institute of Neuroscience, Xuanwu Hospital, Capital Medical University, Beijing 100053 China. E-mail: jixm@ccmu.edu.cn. " These authors contributed equally to this work.

Copyright: () 2018 An H et al. This is an open-access article distributed under the terms of the Creative Commons Attribution License, which permits unrestricted use, distribution, and reproduction in any medium, provided the original author and source are credited. 
PICS is associated with intracranial hemorrhage (ICH) or impaired functional outcomes, thus relevant research on Chinese AIS patients is needed. Some studies reported that PICS was associated with poor clinical outcomes and hemorrhagic transformation [1,3], while others found that extravasation of contrast neither indicated ICH nor impacted functional outcomes [2, 4]. An important reason for these discrepancies may be the different computed tomography (CT) sequences used to evaluate for PICS. Conventional CT scans have limited ability to differentiate contrast material and ICH. Whereas, dualenergy CT (DECT), a relatively new technology, enables reliable differentiation between high attenuation areas related to iodine contrast material extravasation and $\mathrm{ICH}$ on a voxel-by-voxel basis [5].

Different from Western countries, in which AIS is more commonly caused by cardioembolism, the etiology of most AIS patients in Eastern countries, like China, is large artery atherosclerosis (LAA), and approximately half of them have intracranial artery stenosis [6, 7]. However, the incidence of PICS remains unknown, and its effect on functional outcome and ICH in Chinese AIS patients treated with ET is still unclear.

In this study, we aimed to evaluate the incidence of PICS in Chinese AIS patients treated with ET by postoperative DECT scan and investigate whether it is related to functional outcome and $\mathrm{ICH}$.

\section{MATERIALS AND METHODS}

\section{Study population}

The Xuanwu Stroke Center program includes a prospective registry for the study of revascularization therapy (i.e. intravenous thrombolysis and endovascular treatment) for AIS. ET has been applied according to the national guidelines with the intention to start the therapy as soon as possible after symptoms onset. All potential patients were evaluated at admission by a stroke neurologist. Candidates for intravenous thrombolysis or ET underwent emergent non-contrast head CT. CT perfusion or head and neck CT angiography (CTA) was not mandatory. Twelve to 24 hours after ET, all patients underwent a follow-up head CT. For each patient, baseline characteristics, functional outcomes, complications, and follow-up outcomes were documented in the database.

For the present study, all patients treated with ET in our neurovascular unit from January 2013 to February 2017 were selected. Eligibility criteria included 1) AIS patients treated with ET using a second-generation stent retriever device; 2) availability and accessibility of information on baseline data and at least 3-month followup outcome.
The present study was approved by the Ethic Committee of Xuanwu Hospital of Capital Medical University. All patients or their legally authorized representative provided written informed consent at admission to our hospital.

\section{Date collection}

We used the following variables of patients from the database: age, sex, location of occluded artery, time from onset to puncture and recanalization, initial stroke severity assessed by the National Institutes of Health Stroke Scale (NIHSS), initial CT examination evaluated by Alberta Stroke Program Early CT Score (ASPECTS) and posterior circulation ASPECTS (pc-ASPECTS), bridging therapy utilization, risk factors for cerebrovascular diseases, etiology according to the Trial of Org 10172 in Acute Stroke Treatment (TOAST), operation details, ICH, functional outcome at 3 months and long-term follow up assessed by modified Rankin Scale (mRS).

\section{CT imaging}

DECT examination was performed for all patients at 1224 hours after ET while a plain head CT was acquired at $72 \pm 6$ hours post-treatment or whenever an ICH was indicated by clinical evidence. A 64-channel multidetector DECT equipment (Somatom definition FLASH; Siemens) was used for imaging at $90 \mathrm{kV}$ and $150 \mathrm{kV}$ respectively. A three-material decomposition algorithm was applied to differentiate normal brain parenchyma, hemorrhage, and iodine contrast through comparison with unenhanced, virtual images and iodine overlay images. Material with energy-dependent attenuation compatible with iodine was mapped on the iodine overlay image and not on virtual non-contrast (VNC) image, whereas material similar to water (ie, blood) was mapped on the virtual non-contrast image and not on the iodine overlay image $[5,8,9]$. In addition, the differentiation of contrast material extravasation and $\mathrm{ICH}$ was confirmed by comparing the plain CT scan at 72 hours post-treatment with the previous CT scan. Like other neuroimages, all CT images were separately analyzed by a neurologist and a neuroradiologist, and disagreement was resolved by reaching a consensus. If no consensus could be reached, another reviewer made the final decision.

\section{Endpoints}

The primary outcome was the incidence of PICS. PICS was defined as the hyperattenuation areas only seen on the iodine overlay image but not on the virtual non-contrast 
Table 1. Demographic and clinical characteristics of all patients.

\begin{tabular}{|c|c|c|c|c|}
\hline & $\begin{array}{l}\text { All patients } \\
\mathbf{N}=180\end{array}$ & $\begin{array}{l}\text { PICS } \\
\mathbf{N}=\mathbf{5 0}\end{array}$ & $\begin{array}{l}\text { Control } \\
\mathrm{N}=130\end{array}$ & p values \\
\hline Age at onset, mean \pm SD, $y$ & $61.3 \pm 12.8$ & $61.8 \pm 13.1$ & $60.8 \pm 12.4$ & 0.581 \\
\hline Male, n (\%) & $129(72)$ & $30(60)$ & $99(76)$ & $0.031 *$ \\
\hline NIHSS, median (IQR) & $20(14-30)$ & $20(15-33)$ & $21(14-29)$ & 0.615 \\
\hline ASPECTS/pc-ASPECTS, median (IQR) & $9(8-10)$ & $9(8-10)$ & $9(8-10)$ & 0.198 \\
\hline Treatment with IV alteplase, n (\%) & $42(23)$ & $10(20)$ & $32(25)$ & 0.512 \\
\hline OTP time, median (IQR), min & $312(236-378)$ & $297(238-372)$ & $315(232-379)$ & 0.909 \\
\hline OTR time, median (IQR), min & $379(312-434)$ & $403(331-447)$ & $372(284-421)$ & 0.058 \\
\hline \multicolumn{5}{|l|}{ Vascular risk factors, $\mathrm{n}(\%)$} \\
\hline Hypertension & $121(67)$ & $29(58)$ & $92(71)$ & 0.102 \\
\hline DM & $48(26)$ & $12(24)$ & $36(28)$ & 0.616 \\
\hline $\mathrm{AF}$ & 49 (27) & $22(45)$ & $27(21)$ & $0.001 *$ \\
\hline Smoking & $80(44)$ & $19(40)$ & $61(48)$ & 0.317 \\
\hline \multicolumn{5}{|l|}{ Etiology of stroke, n (\%) } \\
\hline LAA & $121(67)$ & $29(58)$ & $92(71)$ & 0.676 \\
\hline Cardioembolism & $43(24)$ & $16(32)$ & $27(21)$ & $0.037 *$ \\
\hline Other & $16(9)$ & $5(10)$ & $11(9)$ & 0.547 \\
\hline \multicolumn{5}{|l|}{ Operational details, n (\%) } \\
\hline General anesthesia & $120(67)$ & $34(68)$ & $86(68)$ & 0.971 \\
\hline Additional intra-arterial thromboly sis & $24(13)$ & $6(12)$ & $18(14)$ & 0.744 \\
\hline Stenting & $53(29)$ & $26(32)$ & $37(29)$ & 0.641 \\
\hline Balloon & $10(6)$ & $2(4)$ & $8(6)$ & 0.840 \\
\hline Tirofiban & $90(50)$ & $22(44)$ & $68(52)$ & 0.318 \\
\hline $\mathrm{TICI}=0$ & $16(9)$ & $5(10)$ & $11(9)$ & 0.547 \\
\hline $\mathrm{TICI}=2 \mathrm{~b} / 3$ & $149(83)$ & $41(82)$ & $108(72)$ & 0.160 \\
\hline
\end{tabular}

PICS, Post-interventional contrast staining; SD, st andard deviation; IQR, interquartile range; NIHSS, National Institutes of Health Stroke Scale; ASPECT, Alberta Stroke Program Early CT score; OTP, time from symptom onset to groin puncture; OTR, time from symptom onset to recanalization; IV, intravenous; TICI, Thrombolysis in Cerebral Infarction; LAA, Large artery atherosclerosis; DM, Diabetes mellitus; AF, Atrial fibrillation.

*A P value less than 0.05 indicates statistical significance.

image of DECT, which was confirmed by comparing plain CT scan at 72 hours post-treatment with the previous CT scan [3]. Secondary outcomes included 1) total ICH; 2) symptomatic ICH (sICH); 3) functional outcome at 3month; and 4) functional outcome at long-term follow up (medium 12 months). Total ICH w as defined ac cording to the Heidelberg Bleeding Classification [10] and SICH was defined according to the ECASS-III study, which classified sICH as any apparent extravascular blood within the brain or cranium that was associated with clinical deterioration (an increase of 4 points or more in NIHSS) [11]. Functional outcome was assessed by modified Ranks scale (mRS), with mRS scores 0-1 indicating no disability, mRS scores 0-2 indicating functional independence, and mRS scores 6 indicating death.

\section{Statistical analysis}

All patients were divided into two groups: PICS and nonPICS (control group) groups. Baseline characteristics and clinical outcomes were compared between the two groups. In multivariate analysis, ICH was adjusted for age, baseline NIHSS, pretreatment with antiplatelet or anticoagulation, hypertension, diabetes mellitus (DM), atrial fibrillation (AF), etiology of stroke, ASPECTS/pcASPECTS, time from onset to groin puncture, additional intra-arterial thrombolysis and treatment with intravenous alteplase. Functional outcomes were adjusted for age, baseline NIHSS, hypertension, DM, AF, etiology of stroke, ASPECTS/pc-ASPECTS, time from onset to groin puncture time, location of stroke (posterior or anterior circulation), symptomatic ICH (sICH), and thrombolysis in cerebral infarction (TICI) scores.

Continuous variables were described by mean \pm SD or median (inter quartile range, IQR). T-test for independent samples or Mann-Whitney U Tests were used to detect differences between the two groups. For binary data, frequency and percentage were used to summarize data, and between-group comparisons were performed via the Chi-square, Continuity Correction or Fisher's exact test as appropriate. All data were analyzed using SPSS 19.0 (IBM Inc.) with the significance level of $\mathrm{p}<0.05$ (twosided). 
Table 2. Demographic and clinical characteristics of anterior circulation stroke.

\begin{tabular}{|c|c|c|c|c|}
\hline & $\begin{array}{l}\text { All patients } \\
\mathrm{N}=118\end{array}$ & $\begin{array}{l}\mathrm{PICS} \\
\mathrm{N}=42\end{array}$ & $\begin{array}{l}\text { Control } \\
N=76\end{array}$ & p -values \\
\hline Age at onset, mean \pm SD & $62.2 \pm 12.8$ & $66.52 \pm 11.39$ & $59.83 \pm 13.05$ & $0.006^{*}$ \\
\hline Male, n (\%) & $77(65)$ & $26(62)$ & $51(67)$ & 0.570 \\
\hline NIHSS, median (IQR) & $17(13-21)$ & $18.5(14-27)$ & $16(13-20)$ & 0.363 \\
\hline ASPECT, median (IQR) & $9(8-10)$ & $9(8-10)$ & $9(8-10)$ & $0.037^{*}$ \\
\hline Treatment with IV alteplase, $\mathrm{n}(\%)$ & $37(31 \%)$ & $10(24)$ & $27(36)$ & 0.189 \\
\hline OTP time, median (IQR) & $286(223-346)$ & $295(238-372)$ & $275.5(197-332)$ & $0.044 *$ \\
\hline \multicolumn{5}{|l|}{ Vascular risk factors, $\mathrm{n}(\%)$} \\
\hline Hypertension & $68(58)$ & $22(52)$ & $46(61)$ & 0.391 \\
\hline DM & $26(22)$ & $9(21)$ & $17(22)$ & 0.906 \\
\hline $\mathrm{AF}$ & $41(35)$ & $19(46)$ & $22(29)$ & 0.075 \\
\hline Smoking & $47(40)$ & $15(38)$ & $32(44)$ & 0.513 \\
\hline \multicolumn{5}{|l|}{ Stroke etiology, n (\%) } \\
\hline LAA & $68(58)$ & $23(55)$ & $45(59)$ & 0.640 \\
\hline Cardioembolism & $38(32)$ & $14(33)$ & $24(32)$ & 0.845 \\
\hline Other & $12(10)$ & $5(12)$ & $7(9)$ & 0.643 \\
\hline \multicolumn{5}{|l|}{ Operation, n (\%) } \\
\hline General anesthesia & $68(58)$ & $27(64)$ & $41(56)$ & 0.394 \\
\hline Additional intra-arterial thrombolysis & $11(9)$ & $3(7)$ & $8(11)$ & 0.545 \\
\hline Stenting & $35(30)$ & $13(31)$ & $22(29)$ & 0.819 \\
\hline Balloon & $6(5)$ & $2(5)$ & $4(5)$ & 0.906 \\
\hline Tirofiban & $56(48)$ & $20(48)$ & $36(47)$ & 0.979 \\
\hline $\mathrm{TICI}=0$ & $12(10)$ & $5(12)$ & $7(9)$ & 0.643 \\
\hline $\mathrm{TICI}=2 \mathrm{~b} / 3$ & $97(82)$ & $34(79)$ & $63(83)$ & 0.606 \\
\hline
\end{tabular}

PICS, Post-interventional contrast staining; SD, st andard deviation; IQR, interquartile range; NIHSS, National Institutes of Health Stroke Scale; ASPECT, Alberta Stroke Program Early CT score; OTP, time from symptom onset to groin puncture; IV, intravenous; TIC, Thrombolysis in Cerebral Infarction; LAA, Large artery atherosclerosis; DM, Diabetes mellitus; AF, Atrial fibrillation. *A P-value less than 0.05 indicates statistical significance.

\section{RESULTS}

A total of 180 AIS patients treated with ET were eligible for this study, including 118 patients $(65.6 \%)$ of anterior circulation stroke (ACS) and 62 patients (34.4\%) of posterior circulation stroke (PCS).

\section{Baseline Data}

The baseline characteristics of all patients are summarized in Table 1. The average age at onset was $61.3 \pm 12$.8-yearold. Thirty of 50 patients $(60 \%)$ in the PICS group and 99 of 130 patients $(76 \%)$ in the control group were male $(\mathrm{p}=0.031)$. Forty-two patients $(23 \%)$ were treated with intravenous alteplase before ET. Median baseline NIHSS score was 20 (14-30), median baseline ASPECTS/pcASPECTS was 9 (8-10), median time from onset to groin puncture and recanalization was 312 (236-378) minutes and 379 (312-434) minutes respectively. Total of 149 patients $(83 \%)$ achieved good or excellent reperfusion (TICI $\geq 2 b)$, and 16 patients $(9 \%)$ failed to achieve any recanalization (TICI $=0)$. Vascular risk factors, etiology of stroke, and operational details are also shown in Table 1. Compared to patients without PICS, more patients with
PICS had a history of AF and an etiology of cardioembolism ( $p<0.05$ each). Demographic and clinical characteristics of the patients with ACS and PCS are summarized in the Table 2 and Table 3, respectively.

\section{Incidence of PICS}

PICS was detected in 50 patients (28\%) (Table 1): 8 of 62 PCS patients (13\%), and 42 of 118 ACS patients (36\%), which was significantly different (Table 2 and 3 , $\mathrm{p}=0.001$ ).

\section{ICH}

In total, ICH was detected in 63 patients (35\%). While 30 of 50 patients $(60 \%)$ experienced ICH in the PICS group, 33 of 130 patients $(25 \%)$ in the control group experienced $\mathrm{ICH}$, which was significantly different $(\mathrm{p}<0.001)$. The incidence of total ICH was also significantly higher in the PICS group than the control group in patients of ACS $(60 \%$ vs. $38 \%, p=0.026)$ and PCS (63\% vs. $7 \%, \mathrm{p}<0.001)$ respectively (Table 4). 
Table 3. Demographic and clinical characteristics of posterior circulation stroke.

\begin{tabular}{|c|c|c|c|c|}
\hline & $\begin{array}{l}\text { All patients } \\
\mathrm{N}=62\end{array}$ & $\begin{array}{l}\text { PICS } \\
\mathrm{N}=8\end{array}$ & $\begin{array}{l}\text { Control } \\
\mathrm{N}=54\end{array}$ & P-values \\
\hline Age at onset, mean \pm SD & $59.7 \pm 12.6$ & $66.38 \pm 9.21$ & $58.72 \pm 12.79$ & 0.109 \\
\hline Male, $n(\%)$ & $52(84)$ & $4(50)$ & $48(89)$ & $0.023^{*}$ \\
\hline NIHSS, median (IQR) & $28(18-32)$ & $33(15.5-35.75)$ & $26(18-30)$ & 0.212 \\
\hline ASPECT, median (IQR) & $9(8-10)$ & $7.5(7-8)$ & $9(8-10)$ & $0.004^{*}$ \\
\hline Treatment with IV alteplase, $\mathrm{n}(\%)$ & $5(8)$ & 0 & $5(9)$ & 0.369 \\
\hline OTP time, median (IQR) & $385(306-445)$ & $317(220-373)$ & $397(314-460)$ & 0.079 \\
\hline \multicolumn{5}{|l|}{ Vascular risk factors, $n$ (\%) } \\
\hline Hypertension & $53(86)$ & $7(88)$ & $46(85)$ & 0.862 \\
\hline DM & $22(36)$ & $3(38)$ & $19(35)$ & 0.898 \\
\hline $\mathrm{AF}$ & $8(13)$ & $3(38)$ & $5(9)$ & $0.026^{*}$ \\
\hline Smoking & $33(53)$ & $4(50)$ & $29(54)$ & 0.845 \\
\hline \multicolumn{5}{|l|}{ S troke etiology, n (\%) } \\
\hline LAA & $53(86)$ & $6(75)$ & $47(87)$ & 0.367 \\
\hline Cardioembolism & $5(8)$ & $2(25)$ & $3(6)$ & 0.059 \\
\hline Other & $4(6)$ & 0 & $4(7)$ & 0.426 \\
\hline \multicolumn{5}{|l|}{ Operation, n (\%) } \\
\hline General anesthesia & $52(84)$ & $7(88)$ & $45(83)$ & 0.765 \\
\hline Additional intra-arterial thromboly sis & $13(21)$ & $3(38)$ & $10(19)$ & 0.218 \\
\hline Stenting & $18(29)$ & $3(38)$ & $15(28)$ & 0.572 \\
\hline Balloon & $4(7)$ & 0 & $4(7.4)$ & 0.426 \\
\hline Tirofiban & $34(55)$ & $2(25)$ & $32(59)$ & 0.069 \\
\hline $\mathrm{TICI}=0$ & $4(7)$ & 0 & $4(7)$ & 0.427 \\
\hline $\mathrm{TICI}=2 \mathrm{~b} / 3$ & $52(84)$ & $7(88)$ & $45(83)$ & 0.765 \\
\hline
\end{tabular}

Nineteen of $180(11 \%)$ patients suffered from sICH, including 10 patients $(8 \%)$ in the control group and 9 patients $(18 \%)$ in the PICS group ( $\mathrm{p}=0.044)$. In patients with PCS, 3 of 8 patients (38\%) with PICS suffered from sICH compared with 1 of 54 patients $(2 \%)$ without PICS $(\mathrm{p}<0.001)$ (Table 4).

\section{Three-month Functional Outcome}

Forty-three of 180 patients (24\%) had no disability at 3months follow up, including 5 patients in the PICS group and 38 patients in the control group, which was significantly different ( $10 \%$ vs. $29 \%, \mathrm{p}=0.007)$ (Table 4$)$. A total of 74 out of 180 patients (41\%) achieved functional independence. Sixteen of 50 patients (32\%) with PICS achieved functional independence, compared to 58 of 130 patients (45\%) without PICS, but no significant difference was detected $(\mathrm{p}=0.123)$. Forty-nine of 180 patients (27\%) died at 3-month follow up, including 17 patients in the PICS group and 32 patients in the control group, which was not significant (34\% vs. $25 \%, \mathrm{p}=0.205)$. However, the mortality of PCS patients was significantly higher in the PICS group than in the control group ( $75 \%$ vs. $30 \%, \mathrm{p}=0.012)$ (Table 4$)$.

\section{Long-term Functional Outcome}

The median length of follow up was 12 (3-26.5) months, and 161 patients finished the long-term follow up (Table 5). For those who died, the follow-up periods were recorded from stroke onset to the time that the subject died. A total of 52 out of 161 patients (32\%) had no disability at long-term follow up, including 7 patients with PICS (16\%) and 44 without PICS (38\%), which was significantly different $(\mathrm{p}=0.006)$. Seventy-four of 161 patients (46\%) achieved functional independence, including 16 patients with PICS (36\%) and 58 patients without PICS (50\%), but no significant difference was detected $(\mathrm{p}=0.099)$. Forty-three of 161 patients (33\%) died at long-term follow up, including 18 patients with PICS (40\%) and 35 patients without PICS (30\%) $(\mathrm{p}=0.234)$.

\section{Multivariate regression analysis}

To determine whether PICS was independently associated with ICH or clinical outcome, multivariate regression analysis was performed. The results revealed that PICS was only independently associated with total ICH (OR 7.38, 95\% CI 1.66-32.90; $\mathrm{p}=0.009)$. However, PICS did not have an impact on sICH, 3-months func tional outc ome 
or long-term functional outcome ( $>00.1$ each). Additionally, there was no correlation between PICS and mortality when controlling for other factors (Table 6). All factors that were included in the multivariate analysis to predict ICH are summarized in Table 7. Only PICS and additional intra-arterial thrombolysis affected ICH significantly.

Table 4. Intracerebral hemorrhage and 3-month follow up.

\begin{tabular}{|c|c|c|c|c|c|c|c|c|c|c|}
\hline & \multicolumn{4}{|c|}{$\begin{array}{c}\text { All patients, } \\
\mathbf{N}=180\end{array}$} & \multicolumn{3}{|c|}{$\begin{array}{c}\text { Anterior circulation } \\
\text { stroke, } N=118\end{array}$} & \multicolumn{3}{|c|}{$\begin{array}{c}\text { Posterior circulation } \\
\text { stroke, } \mathrm{N}=62\end{array}$} \\
\hline & All & $\begin{array}{l}\mathrm{PICS} \\
\mathrm{N}=50\end{array}$ & $\begin{array}{l}\text { Control } \\
\mathrm{N}=130\end{array}$ & p-values & $\begin{array}{l}\mathrm{PICS} \\
\mathrm{N}=42\end{array}$ & $\begin{array}{l}\text { Control } \\
\mathrm{N}=76\end{array}$ & $\mathrm{p}$-values & $\begin{array}{l}\mathrm{PICS} \\
\mathrm{N}=8\end{array}$ & $\begin{array}{l}\text { Control } \\
\mathrm{N}=54\end{array}$ & p-values \\
\hline $\begin{array}{l}\text { Total ICH, n (\%) } \\
\text { sICH, n (\%) } \\
\text { 3-month mRS, n (\%) }\end{array}$ & $\begin{array}{c}63(35) \\
19(11)\end{array}$ & $\begin{array}{c}30(60) \\
9(18)\end{array}$ & $\begin{array}{c}33(25) \\
10(8)\end{array}$ & $\begin{array}{c}<0.001^{*} \\
0.044^{*}\end{array}$ & $\begin{array}{c}25(60) \\
6(14)\end{array}$ & $\begin{array}{c}29(38) \\
9(12)\end{array}$ & $\begin{array}{c}0.026^{*} \\
0.703\end{array}$ & $\begin{array}{l}5(63) \\
3(38)\end{array}$ & $\begin{array}{l}4(7) \\
1(2)\end{array}$ & $\begin{array}{l}<0.001^{*} \\
<0.001^{*}\end{array}$ \\
\hline $\begin{array}{l}\mathrm{mRS}, 0-1 \\
\mathrm{mRS}, 0-2 \\
\mathrm{mRS}, 6\end{array}$ & $\begin{array}{l}43(24) \\
74(41) \\
49(27)\end{array}$ & $\begin{array}{c}5(10) \\
16(32) \\
17(34)\end{array}$ & $\begin{array}{l}38(29) \\
58(45) \\
32(25)\end{array}$ & $\begin{array}{c}0.007^{*} \\
0.123 \\
0.205\end{array}$ & $\begin{array}{c}5(12) \\
15(36) \\
11(26)\end{array}$ & $\begin{array}{l}29(38) \\
43(56) \\
16(21)\end{array}$ & $\begin{array}{c}0.003 * \\
0.030 \\
0.525\end{array}$ & $\begin{array}{c}0 \\
1(13) \\
6(75)\end{array}$ & $\begin{array}{c}9(17) \\
15(28) \\
16(30)\end{array}$ & $\begin{array}{c}0.212 \\
0.357 \\
0.012 *\end{array}$ \\
\hline
\end{tabular}

PICS, Post-interventional contrast st aining; ICH, Intracranial Hemorrhage; sICH, symptomatic Intracranial Hemorrhage; mRS, modified Ranks Scale.

*A P value less than 0.05 indicates statistical significance.

\section{DISCUSSION}

In this study, we found that PICS was detected in over one-fourth of AIS patients treated by ET, and it was an independent predicator for total ICH. However, PICS may not be associated with the functional outcome and sICH. Collectively, these results support the clinical relevance of DE-CT to identify early BBB disruption in patients with AIS receiving ET. These results also hint that PICS without final ICH may only require routine follow-up.

The incidence of PICS in our cases was approximately $28 \%$, which was lower than previous studies $(31.2 \%$ and $60.3 \%)[1,4]$. This discrepancy could be attributed to several factors. Firstly, contrast staining appears to be a transient phenomenon, thus it is more likely to be detected if the time interval between the ET and the follow-up CT is short [2]. Secondly, previous studies selected different definitions for PICS and CT sequence, which potentially conflated contrast staining and ICH $[1,4]$. Thirdly, AIS caused by cardioembolism are less likely in the Chinese population than in western populations [12]. The most common cause of AIS in our subjects was LAA $(67 \%)$, while only $24 \%$ patients are cardioembolism. Based on our results, LAA patients were just as likely to develop PICS as other patients $(\mathrm{p}=0.676)$, however cardioembolism patients were more likely to develop PICS than not $(\mathrm{p}=0.037)$. The higher proportion of LAA patients in this study may partially account for the lower rates of PICS than previously reported.

Table 5. Long-term outcome.

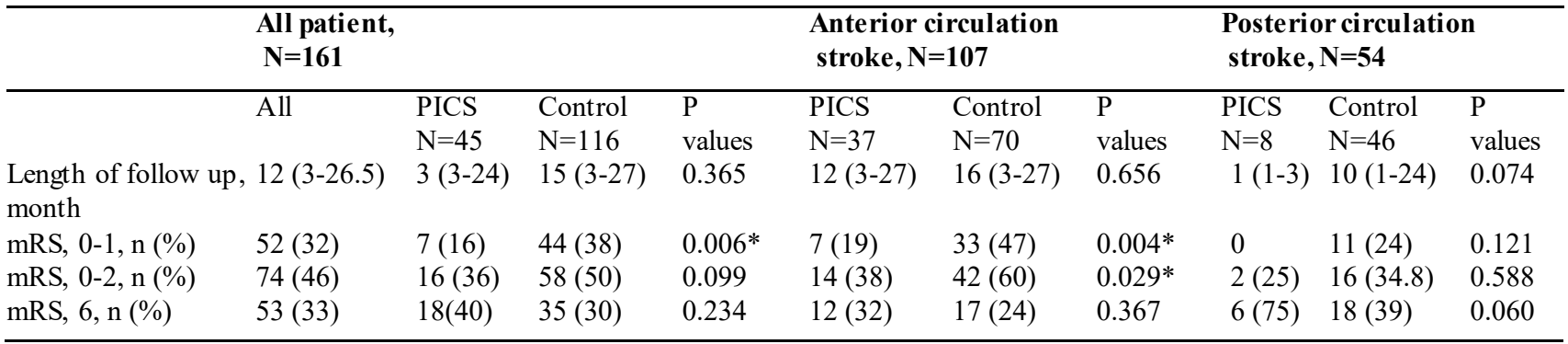

PICS, Post-interventional contrast staining; mRS, modified Ranks Scale. *A P-value less than 0.05 indicates st atistical significance

Interestingly, we found that PICS was more frequently detected in patients with ACS than in patients with PCS. Several studies, focusing on the neuroanatomy and physiology, may provide insight into this finding. Firstly, previous studies showed that the incidence of PICS was associated with infarct volume [2], and the infarct volume in infratentorial strokes is often smaller than that of supratentorial strokes. Secondly, previous studies have identified that the posterior circulation has a greater ischemic tolerance, and BBB disruption may be delayed in the posterior circulation after stroke onset when compared to the anterior circulation [13-17]. 
Table 6. Multivariate regression analysis of the effects of PICS on ICH and functional outcome.

\begin{tabular}{lcccc}
\hline \multicolumn{1}{c}{ Outcome } & Adjusted P value & OR & \multicolumn{2}{c}{ 95\% CIs } \\
\cline { 3 - 5 } & & & Lower limit & Upper limit \\
\hline Total ICH $^{\#}$ & $0.018^{*}$ & 7.38 & 1.66 & 32.90 \\
sICH $^{\#}$ & 0.994 & -- & - & -- \\
3-months mRS, 0-1 & 0.522 & 0.50 & 0.06 & 4.182 \\
3-months mRS, 0-2 & 0.451 & 2.03 & 0.32 & 12.77 \\
3-months mortality & 0.937 & 1.07 & 0.18 & 6.46 \\
PCS mortality $\$$ & 0.147 & 9.48 & 0.45 & 198.30 \\
Long-term mRS, 0-1 $^{\dagger}$ & 0.141 & 0.22 & 0.03 & 1.65 \\
Long-term mRS, 0-2 $^{\dagger}$ & 0.181 & 3.30 & 0.58 & 17.65 \\
Long-term mortality $^{\dagger}$ & 0.122 & 0.72 & 0.11 & 4.61 \\
\hline
\end{tabular}

\#Adjusted for age, baseline NIHSS, pretreatment with antiplatelet or anticoagulation, hypertension, diabetes mellitus, atrial fibrillation, etiology of stroke, ASPECTS/pc-ASPECTS, time from onset to groin puncture, treatment with additional intra-arterial thrombolysis, and treatment with intravenous alt eplase. $\uparrow$ Adjusted for age, baseline NIHSS, hypertension, diabetes mellitus, atrial fibrillation, etiology of stroke, ASPECTS/pc-ASPECTS, time from onset to groin puncture, location of stroke (posterior or anterior circulation), sICH, and thrombolysis in cerebral infarction (TICI) scores.§Adjusted for age, baseline NIHSS, hypertension, diabetes mellitus, atrial fibrillation, etiology of stroke, ASPECTS/pc-ASPECTS, time from onset to groin puncture, sICH, and thrombolysis in cerebral infarction (TICI) scores. *A P value less than 0.05 indicates statistical significance.

Thirdly, PCS tend to have better collateral circulation, which is critical to rescue the ischemic penumbra. Finally, both vasoreactivity and autoregulation differ between the anterior and posterior circulations. The insensitivity to autoregulation in PCS may be a factor in limiting the amount of contrast extravasation into the parenchyma [1820]. Our study revealed that PICS was associated with $\mathrm{ICH}$, which supported the concept that both PICS and ICH are successive stages of $\mathrm{BBB}$ damage [21]. When the ischemic injury is limited to the endothelial cells, contrast staining may be composed solely of contrast medium.
However, when injury extends to the basal lamina, contrast staining may be associated with some degree of $\mathrm{ICH}$ [22]. Early reperfusion is crucial to prevent damage to the BBB and avoid ICH. This is also why PICS was associated with $\mathrm{ICH}$ and poor clinical outcomes in previous studies of intra-arterial thrombolysis which had relatively low recanalization rates $[23,24]$. This finding was further supported by our data which showed that the onset to recanalization times were longer in the PICS group compared to the control.

Table 7. Multivariate analys is of ICH.

\begin{tabular}{llll}
\hline Variates & OR & $\mathbf{9 5 \%}$ CI & P-value \\
\hline Age & 0.969 & $0.91-1.03$ & 0.296 \\
Hypertension & 3.165 & $0.49-20.44$ & 0.226 \\
DM & 0.329 & $0.05-2.09$ & 0.238 \\
AF & 4.935 & $0.62-39.18$ & 0.131 \\
NIHSS & 0.999 & $0.92-1.09$ & 0.984 \\
Pre-Antiplatelet/Anticoagulation & 0.342 & $0.06-1.88$ & 0.218 \\
Etiology of stroke & 1.736 & $0.87-3.48$ & 0.120 \\
ASPECT/pc-ASPECTS & 0.590 & $0.36-0.97$ & 0.036 \\
OTP time & 0.997 & $0.99-1.00$ & 0.401 \\
Additional intra-arterial thrombolysis & 7.581 & $1.16-49.74$ & $0.035^{*}$ \\
Treatment with IV alteplase & 2.625 & $0.51-13.55$ & 0.249 \\
PICS & 6.370 & $1.37-29.70$ & $0.018^{*}$ \\
\hline
\end{tabular}

PICS, Post-interventional contrast st aining; NIHSS, National Institutes of Health Stroke Scale; ASPECT, Alberta Stroke Program Early CT score; OTP, time from symptom onset to groin puncture; IV, intravenous; DM, Diabetes mellitus; AF, Atrial fibrillation. *A P value less than 0.05 indicates statistical significance. 
Contrary to Arturo's study which demonstrated that PICS was associated with poor clinical outcomes [3], our study found that PICS did not affect functional outcomes or mortality. This discrepancy may be attributed to various stroke etiologies in different ethnicities. Compared to the west, LAA is reported to be the more likely etiology for stroke in our Chinese population [7, 25]. PICS patients have higher percentage of cardioembolism events compared to LAA. This is because the former causes emergent large vessels occlusion with poor collateral circulation, which may lead to serious damage to the BBB $[26,27]$. Approximately half of the acute ischemic strokes were due to cardioembolism in Arturo's study, whereas less than one fourth were due to this etiology in our study. Additionally, according to our results, mortality in PICS group was higher than the control group in PCS patients. However, this difference was not apparent after adjusting for relative factors especially for the pc-ASPECT score. This score was traditionally known as a predictor for the infarct volume and clinical outcome, with lower scores indicating larger infarct volume and worse clinical outcomes [28, 29].

Limitations of this study include small sample size which limits the utility of our conclusion. Additionally, this was an observational study and the outcome assessment was not blinded for either the baseline characteristics or treatment. Potential variables including additional use of intra-arterial thrombolysis and various etiologies of stroke might also have confounded out study results.

\section{Conclusion}

PICS is a common phenomenon in Chinese AIS patients treated with ET. Even though PICS is associated with high incidence of $\mathrm{ICH}$, no correlation to sICH or functional outcome was evident in our study. Additional, large-scale studies are needed to confirm our results and explore their influence on postoperative therapeutic guidelines.

\section{Acknowledgments}

This work was partially supported by Science and technology Beijing one hundred leading talent training project (Z141107001514006); Beijing Municipal Administration of Hospitals' Mission Plan (SML 20150802).

\section{References}

[1] RouchaudA, Pistocchi S, Blanc R, Enqrand N, Bartolini B, Piotin M (2014). Predictive value of flat-panelCT for haemorrhagic transformations in patients with acute stroke treated with thrombectomy. J Neurointerv Surg,
6:139-43

[2] Nikoubashman O, Reich A, Gindullis M, et al (2014). Clinical significance of post-interventional cerebral hyperdensities after endovascular mechanical thrombectomy in acute ischaemic stroke. Neuroradiology, 56:41-50.

[3] Renú A, Amaro S, Laredo C, Román LS, Llull L, Lopez A, Urra X, Blasco J, Oleaga L, Chamorro Á (2015). Relevance of blood-brain barrier disruption after endovascular treatment of is chemic stroke: dual-energy computed tomographic study. Stroke, 46:673-79.

[4] Parrilla G, Garcia-Villalba B, Espinosa de Rueda M, Zamarro J, Carrión E, Hernández-Fernández F, Martín J, Hernández-Clares R, Morales A, Moreno A (2012). Hemorrhage/contrast staining areas after mechanical intra-arterial thrombectomy in acute ischemic stroke: imaging findings and clinical significance. AJNR AmJ Neuroradiol, 33:1791-96.

[5] $\mathrm{Hu}$ R, Padole A, Gupta R (2017). Dual-Energy Computed Tomographic Applications for Differentiation of Intracranial Hemorrhage, Calcium, and Iodine. Neuroimaging Clin N Am, 27:401-09.

[6] Li F, Yang L, Yang R, Xu W, Chen FP, Li N, Zhang JB (2017). Ischemic Stroke in Young Adults of Northem China: Characteristics and Risk Factors for Recurrence. Eur Neurol, 77:115-22.

[7] Kernan WN, Ovbiagele B, Black HR, et al (2014). Guidelines for the prevention of stroke in patients with stroke and transient ischemic attack: a guideline for healthcare professionals from the American Heart Association/American Stroke Association. Stroke, 45: 2160-236.

[8] Tijssen MP, Hofman PA, Stadler AA, van Zwam W, de Graaf R, van Oostenbrugge RJ, Klotz E, Wildberger JE, Postma AA (2014). The role of dual energy CT in differentiating between brain haemorrhage and contrast medium after mechanical revascularisation in acute ischaemic stroke. Eur Radiol, 24:834-40.

[9] Liu X, Yu L, Primak AN, McCollough CH (2009). Quantitative imaging of element composition and mass fraction using dual-energy CT: three-material decomposition. Med Phys, 36:1602-09.

[10] von Kummer R, Broderick JP, Campbell BC, et al (2015). The heidelberg bleeding classification: classification of bleeding events after ischemic stroke and reperfusion therapy. Stroke, 46:2981-86.

[11] Hacke W, Kaste M, Bluhmki E, Brozman M, et al(2008). Thrombolys is with alteplase 3 to 4.5 hours after acute ischemic stroke. N Engl J Med, 359:1317-29.

[12] Zhao W, Che R, Shang S, et al (2017). Low-dose Tirofiban Improves Functional Outcome in Acute Ischemic Stroke Patients Treated With Endova scular Thrombectomy. Stroke, 48:3289-94.

[13] Bang OY, Saver JL, Kim SJ, Kim GM, Chung CS, Ovbiagele B Lee KH, Liebeskind DS; UCLASamsung Stroke Collaborators (2011). Collateral flow averts hemorrhagic transformation after endovascular therapy for acute ischemic stroke. Stroke, 42:2235-39.

[14] Menon BK, O'Brien B, Bivard A, Spratt NJ, Demchuk AM, Miteff F, Lu X, Levi C, Parsons MW (2013). 
Ass essment of leptomeningeal collaterals using dynamic CT angiography in patients with acute is chemic stroke. $\mathrm{J}$ Cereb Blood Flow Metab, 33:365-71.

[15] Lindgren A, Norrving B, Rudling O, Johansson BB (1994). Comparis on of clinical and neuroradiological findings in first-ever stroke. A population-based study. Stroke, 25:1371-77.

[16] Bang OY, Saver JL, Alger JR, Shah SH, Buck BH, Starkman S, Ovbiagele B, Liebeskind DS; UCLA MRI Permeability Investigators (2009). Patterns and predictors of blood-brain barrier permeability derangements in acute ischemic stroke. Stroke, 40:45461.

[17] Lee M, Saver JL, Alger JR, Hao Q, et al (2011). Bloodbrain barrier permeability derangements in posterior circulation ischemic stroke: frequency and relation to hemorrhagic trans formation. J Neurol Sci, 313:142-46.

[18] Haubrich C, Wendt A, Diehl RR, Klötzsch C (2004). Dynamic autoregulation testing in the posterior cerebral artery. Stroke, 2004. 35:848-52.

[19] McKinney AM, Sarikaya B, Gustafs on C, Truwit CL (2012). Detection of microhemorrhage in posterior reversible encephalopathy syndrome using susceptibility-weighted imaging. AJNR Am J Neuroradiol, 33:896-903.

[20] Covarrubias DJ, Luetmer PH, Campeau NG (2002). Posterior reversible encephalopathy syndrome: prognostic utility of quantitative diffusion-weighted MR images. AJNR Am J Neuroradiol, 23:1038-48.

[21] Nakano S, Iseda T, Kawano H, Yoneyama T, Ikeda T, Wakisaka S (2001). Parenchymal hyperdensity on computed tomography after intra-arterial reperfusion therapy for acute middle cerebral artery occlusionIncidence and clinical significance. Stroke, 32:2042-48.

[22] Desilles JP, Rouchaud A, Labreuche J, Mesequer E, Laissy JP, Serfaty JM, Lapergue B, et al (2013). Bloodbrain barrier disruption is associated with increased mortality after endoas cular therapy. Neurology, 80:84451.

[23] Jang YM, Lee DH, Kim HS, Ryu CW, Lee JH, Choi CG, Kim SJ, Suh DC (2006). The fate of high-density lesions on the non-contrastCT obtained immediately after intraarterial thrombolys is in is chemic stroke patients. Korean J Radiol, 7:221-28.

[24] Yoon W, Seo JJ, Kim JK, Cho KH, Park JG, Kang HK (2004). Contrast enhancement and contrast extravasation on computed tomography after intra-arterial thrombolys is in patients with acute ischemic stroke. Stroke, 35: 876-81.

[25] Zhao W, Shang S, Li C, Wu L, Wu C, Chen J, Song H, Zhang H, Zhang Y, Duan J, Feng W, Ji X (2018). Long-term outcomes of acute is chemic stroke patients treated with endovascular thrombectomy: A real-world experience. J Neurol Sci, 390: 77-83.

[26] O'Carroll CB, Barrett KM (2017). Cardioembolic Stroke. Continuum, 23:111-32.

[27] Wang XG, Zhang LQ, Liao XL, et al (2015). Unfavorable Outcome of Thrombolys is in Chinese Patients with Cardioembolic Stroke: a Prospective Cohort Study. CNS Neurosci Ther, 21:657-61.
[28] Nagel S, Wang X, Carcel C, Robins on T, Lindley RI, Chalmers J, et al (2018). Clinical Utility of Electronic Alberta Stroke Program Early Computed Tomography Score Software in the ENCHANTED Trial Database. Stroke, 49:1407-11.29.

[29] Aviv RI, Mandelcorn J, Chakraborty S, Gladstone D, Malham S, Tomlinson G, Fox AJ, Symons S (2007). Alberta Stroke Program Early CT Scoring of CT perfusion in early stroke visualization and as sessment. AJNR Am J Neuroradiol, 28:1975-80. 